\title{
Narrativas y resiliencia. Las historias de vida como mediación metodológica para reconstruir la existencia herida*
}

\section{Narratives and Resilience Life Stories as a Methodological Mediation to Rebuild the Wounded Existence}

\author{
Luis Fernando Granados ${ }^{1}$ CVLAC - Sara Victoria Alvarado ${ }^{2}$ CvLAC $_{-}$Jaime Carmona \\ 'Pontificia Universidad Javeriana, ${ }^{2,3}$ Universidad de Manizales
}

Colombia

Fecha correspondencia:

Recibido: febrero 2 de 2016.

Aceptado: noviembre 11 de 2016.

Forma de citar:

Granados, L., Alvarado, S. \&

Carmona, J. (2016). Narrativas y

resiliencia. Las historias de vida

como mediación metodológica para

reconstruir la existencia herida.

Rev. CES Psicol., 10(1), 1-20.

\section{Open access \\ (c) Copyright}

Licencia creative commons

Etica de publicaciones

Revisión por pares

Gestión Open Journal System

DOI: http://dx.doi.org/10.21615/

cesp.10.1.1

ISSN: 2011-3080

Sobre el artículo:

Este artículo de reflexión derivado de un trabajo de investigación, tiene como procedencia y está inscrito, en el trabajo de investigación sobre "resiliencia y lo políitico", que se está realizando en el marco del Doctorado en Ciencias

\section{Resumen}

Este artículo busca poner en diálogo la categoría de resiliencia con el enfoque narrativo, desde un acercamiento a las historias de vida como mediación metodológica cualitativa de particular significación, para leer de manera comprensiva lo que acontece a los sujetos cuando relatan la vida contando historias, reconociendo que el accenso a la humanidad como construcción subjetiva pasa por esa capacidad para relatar la propia vida, atravesada por experiencias límites. La atención se pone en las mismas historias de vida como mediación y su significación para la investigación en resiliencia. El método de trabajo se apoyó en una revisión documental, con un trabajo de análisis categorial, buscando elementos teóricos y metodológicos que fortalezcan la investigación en resiliencia desde el enfoque narrativo.

Palabras clave: Resiliencia, Narrativa, Mediación Cualitativa, Método Biográfico, Método Autobiográfico, Historias de Vida, Relatos de Vida, Adversidad.

\section{Abstract}

This article seeks to category resilience dialogue with the narrative approach from an approach to life stories and qualitative methodological mediation of particular meaning to read comprehensively what happens when subjects relate the life telling stories. Recognizing that accenso humanity as subjective construction, goes through that ability to relate one's life, crossed by boundaries experiences. The focus is placed on the same stories of life as mediation and its significance for research on resilience. The working method involves a literature review and a categorical analysis work, looking for theoretical and methodological elements that strengthen resilience research from the narrative approach.

Keywords: Resilience, Narrative, Qualitative Mediation, Biographical Method, Autobiographical Method, Life Stories, Stories of Life, Adversity. 
Pág 2

Sociales Ninez y Juventud del CINDE de la Universidad de Manizales. El trabajo doctoral a partir de las narrativas de los estudiantes del programa "Ser Pilo Paga" de la Pontificia Universidad Javeriana de Cali, tiene como objetivo poner en tensión y dialogo la categoría de la resiliencia con lo político, desde la experiencia narrada por los estudiantes en sus tránsitos de la adversidad en la vida, a la acción conjunta y al compromiso solidario, que deja entrever la acción política en búsqueda de la construcción de sentidos de vida y la lucha por una vida digna. El artículo se centra en las historias de vida como poderosa mediación metodológica y lugar desde donde se lee, se comprende y se asume la existencia herida para su trasformación.

Sobre los autores:

1. Magíster en Educación de la Pontificia Universidad Javeriana, Estudiante del Doctorado en Ciencias Sociales, Niñez y Juventud.

2. Postdoctorado Universidad de Manizales, CINDE, Universidad Católica de Sao Paulo, Clacso; Doctora en Educación de Nova University - CINDE; Directora del Doctorado en Ciencias Sociales con énfasis en Niñez y Juventud, de la Universidad de Manizales y el Cinde, Colombia.

3. Doctor en Psicología Social, Universidad Complutense de Madrid; Magíster en Ciencias Sociales, Universidad de Antioquia; Magíster en Educación, Universidad Católica de Manizales; Psicólogo Social, Universidad Autónoma de Monterrey; Comunicador Social y Periodista, Universidad de Antioquia; Coordinador de Investigaciones, Centro de Estudios Avanzados en Niñez y Juventud, Universidad de Manizales - CINDE.
De siempre ha sido conocido y se ha dicho que la vida tiene que ver con la narración; hablamos de la historia de una vida para caracterizar el intervalo entre nacimiento y muerte. Y, con todo, esta asimilación de la vida a la historia no es automática; se trata, incluso, de una idea trivial que es necesario someter, antes de nada, a una duda crítica.

Esta duda es el resultado de todo el conocimiento adquirido en las décadas pasadas en relación al relato y a la actividad narrativa, un saber que parece alejar el relato de la vida en tanto que vivida y que confina al relato en el campo de la ficción.

Paul Ricoeur. "La vida, un relato en búsqueda de narrador".

\section{Introducción}

La resiliencia tiene que ver con contar historias, con la manera como los sujetos narran historias a partir de experiencias vitales fruto del afrontamiento de la adversidad. El accenso a la humanidad como construcción subjetiva pasa por esa capacidad para relatar la propia vida, atravesada por experiencias límites, en las que se configuran tramas densas, inmersas en contextos sociales, económicos y culturales complejos por su diversidad y pluralidad. Vivir es relatar. No es posible una vida auténtica sin relato. El relato es una forma de inteligibilidad, una forma en que el sujeto se comprende a sí mismo y el mundo social en el que habita (Delory-Momberger. 2015). Existe sin duda una relación estrecha entre vivir y narrar, entre la trama del vivir y la búsqueda de sentido (Ricoeur, 2006).

Los seres humanos dan sentido a su mundo cotidiano contando historias sobre sí mismos (Bruner, 1997). Ese contar historias va configurando su identidad, precisamente en el trabajo de relatar lo que les pasa y lo que los sujetos hacen con lo que les pasa. El sujeto se constituye y se crea cuando está relatando su propia vida. Allí se auto-interpreta buscando ordenar y dar significado a la propia existencia (Bruner, 2003).

La resiliencia, como categoría comprensiva de procesos de humanización, está reclamando una lectura narrativa que dé cuenta de los tránsitos de un sujeto que, en situaciones de adversidad, tiene la capacidad para narrar su historia. Al contar historias, los sujetos se adentran en escenarios evocativos y creativos.

Los sujetos, desde una narrativa resiliente, tienen la capacidad para trabajar y aprender de su propia auto-percepción, ayudados por los intercambios intersubjetivos que establecen con otros y que les permiten fortalecerse para afrontar la adversidad. El trabajo narrativo entiende al ser humano no como un dato determinado o acabado, sino como un proceso de vida, un proyecto que se hace, que se va expresando, desplegando y significando, cuando los sujetos son capaces de contar lo vivido como acontecimiento significativo y transformador.

Este artículo recoge en primera instancia una mirada crítica sobre la categoría de resiliencia y su tránsito en las Ciencias Sociales. Desde una visión de la resiliencia como capacidad para construir vida en medio de la adversidad, reconoce el valor que para este tejido vital tienen las narrativas propias del ámbito autobiográfico, profundizando en las historias de vida y su significación para la comprensión de la resiliencia. El artículo finaliza instalando un diálogo constructivo entre el trabajo con narrativas y el desarrollo de la resiliencia como proceso de humanización.

\section{Resiliencia: entre el "resistir" y el "rehacerse"}

Según algunos autores, la resiliencia ha llegado para darle un sentido y una significación a la adversidad (Vanistendael \& Lecomte, 2002). En este sentido, se inscribe 
La resiliencia a partir de un enfoque más comunitario y político, busca desplegar en los sujetos su capacidad de agenciamiento y su potencial para desatar acciones personales, comunitarias y colectivas que incidan en la reconstrucción del tejido social y sus trasformaciones. como las narrativas, en un horizonte hermenéutico que busca comprender la significación que dichas experiencias ofrecen para la construcción de la propia vida entendida como proyecto.

El término procede del vocablo latino resilio (Kotliarenco, Cáceres, \& Fontecilla, 1997). En la física designa la capacidad de un cuerpo para resistir una fuerza y volver a su estado original. En el ámbito de lo humano, la resiliencia está indicando la dinámica de un proceso que tiene que ver con la capacidad de los sujetos de resistir y rehacerse en medio de conflictos y tensiones, para salir hacia adelante fortalecidos.

Desde la perspectiva de Puig G \& Rubio, L. (2011) la resiliencia es un concepto en permanente evolución, que parte de hechos observables en las realidades humanas, que reconoce el valor de una actitud vital y de un pensamiento constructivo, que valora las posibilidades de las personas y sus redes de apoyo, que incorpora la adversidad como parte del ciclo vital, que asume una posición ética de respeto y de sensibilidad por los demás y que está buscando dotar de sentido y significación a la experiencia humana. Desde su comprensión entienden que las definiciones actuales de resiliencia están enfatizando rasgos propios de lo que ellos llaman una persona resiliente.

Las principales definiciones enfatizan las características de la persona resiliente: habilidad, adaptabilidad, baja susceptibilidad, enfrentamiento efectivo, capacidad, resistencia a la destrucción, conductas vitales positivas, temperamento especial, habilidades cognitivas, todas desplegadas frente a las situaciones vitales adversas, estresantes, etc., que les permite atravesarlas y superarlas (Puig \& Rubio, 2011, p. 43).

La perspectiva clásica de la resiliencia habla de ella como un proceso de desarrollo positivo a pesar de vivir en ambientes insanos, enfatizando la capacidad humana para responder y adaptarse a pesar de altas predicciones de riesgo. La resiliencia integra procesos propios del sujeto y atributos protectores del ambiente familiar, social y cultural, que, en los procesos de interacción humana, fortalecen y potencian la capacidad de los sujetos y las comunidades para el afrontamiento de las tensiones y contradicciones que surgen en el camino de la vida.

La resiliencia es la capacidad de una persona o de un grupo para desarrollarse bien, para seguir proyectándose en el futuro a pesar de acontecimientos desestabilizadores, de condiciones de vida difíciles y de traumas a veces graves... La resiliencia nunca es absoluta, total, lograda para siempre. Es una capacidad que resulta de un proceso dinámico, evolutivo, en la que la importancia de un trauma puede superar los recursos del sujeto; varía según las circunstancias, la naturaleza del trauma, el contexto y la etapa de la vida; puede expresarse de modos muy diversos según la cultura (Manciaux, 2003, pp. 22-23).

Partiendo de una mirada más reflexiva propia del campo de las Ciencias Sociales, se entiende que la resiliencia va más allá del simple resistir o salir fortalecido en medio del caos, el trauma o la situación de adversidad. La resiliencia a partir de un enfoque más comunitario y político, busca desplegar en los sujetos su capacidad de agenciamiento y su potencial para desatar acciones personales, comunitarias y colectivas que incidan en la reconstrucción del tejido social y sus trasformaciones.

La resiliencia desde una mirada crítica, está llamada a develar situaciones en las que la adversidad esconde tensiones y conflictos propios de sociedades excluyentes 
e inequitativas. La resiliencia potencia la capacidad para de-construir y reconstruir tramas y sentidos, favoreciendo prácticas intersubjetivas en las que los sujetos puedan desplegar lo que algunos autores han llamado la subjetividad política.

El desarrollo humano, centrado en la expansión de las capacidades, de las libertades y de las posibilidades de los niños, niñas y jóvenes como actores protagonistas en la construcción de dinámicas y sentidos de ciudadanía y de la construcción de paz, implica un proceso de formación ciudadana y de subjetividad que se inicia en el reconocimiento de estos sujetos como seres inacabados en permanente construcción y significación, es decir, comprende que el ser humano es un ser en permanente tensión, cuya construcción intersubjetiva está mediada por procesos de socialización mediante los cuales nos dotamos de humanidad (Alvarado, 2014, p. 23).

Desde la lógica interpretativa de este trabajo, la resiliencia se presenta como una posibilidad para que los sujetos puedan leer, entender, comprender y transformar la adversidad, permitiendo el descubrimiento de aquello que ayuda a no quedarse atrapado en lo que pasó, más bien abrir el camino para construir, preservar o reconstruir una nueva dinámica de vida que se pregunta por el ¿para qué? de lo que pasó.

Cuando el dolor es demasiado fuerte, nos vemos sometidos a su percepción. Sufrimos. Pero apenas podemos tomar un poco de distancia, apenas podemos convertirlo en representación teatral, la desdicha se hace soportable, o más bien la memoria de la desdicha se metamorfosea en risa o en obra de arte... nuestra historia no es un destino. Nada queda escrito para siempre... la carencia precoz crea una vulnerabilidad momentánea, que las experiencias afectivas y sociales podrán reparar o agravar. En este sentido la resiliencia constituye un proceso natural en el que lo que somos en un momento dado necesariamente debe entretejerse con los medios ecológicos, afectivos y verbales... Basta con que haya un sólo punto de apoyo para que la edificación pueda continuar (Cyrulnik, 2001, pp. 13-15).

Es en esta capacidad de distanciamiento para la re-significación del acontecimiento vital, en esta posibilidad de tejer un nuevo comienzo con los hilos adoloridos por el sufrimiento, donde se sitúa la potente relación entre la resiliencia y el lenguaje narrativo que permite otorgarle la palabra a los sujetos como protagonistas en la búsqueda de un sentido de vida, sobre todo al pasar por situaciones densas y difíciles.

La resiliencia pone en tensión la experiencia cruda del vivir, no se puede soslayar el sufrimiento y sus causas, como la responsabilidad de los agresores, pero cuando el sobreviviente es capaz de transitar en la elaboración de la vida por la vida del sentido, el agresor pierde protagonismo y lo que surge es una nueva narrativa que potencia la voz de los que han sido sistemáticamente silenciados. El sujeto emerge en su subjetividad posicionando las emergencias de un nuevo nacimiento que hace renacer la esperanza.

\section{Las narrativas en el ámbito autobiográfico}

Es un hecho que las Ciencias Sociales se inclinan hoy con mayor recurrencia a escuchar la voz y el testimonio de los sujetos, reconociéndolos como auténticos protagonistas o actores sociales (Arfuch, 2010). Se trata de lo que se ha llamado una "vuelta al sujeto" como lugar epistémico, de donde surge la reconfiguración de la 
El giro subjetivo de esta época está hablando de la cercanía, proximidad y la veracidad del testimonio como fuentes para comprender al sujeto que, por el trabajo narrativo, ha podido dialogar con ese otro que se encuentra inmerso en su intimidad, que emerge gracias al lenguaje y a la experiencia de la otredad que es el reconocimiento de la intersubjetividad que lo constituye. Las narrativas son en últimas una extensión de los enfoques interpretativos en Ciencias Sociales (Escalante, 2013). subjetividad. Las narrativas del yo se están haciendo presentes: biografía, autobiografía, diarios personales, cartas, rostros, voces y cuerpos nos están hablando de lo que han vivido, lo que han visto y lo que han padecido (Arfuch, 2010a). La identidad de un sujeto tiene que ver con la capacidad que tiene el mismo sujeto para narrar su historia desde su experiencia autobiográfica (Gergen, 1996).

Esa "vuelta al sujeto" no es solamente un retorno al individuo en su capacidad de saber, sentir y actuar, sino también al sujeto como realidad social, como constructor de cultura (Loureiro, 1991). La narrativa como acción del sujeto sobre su propia construcción identitaria tiene que ver con su temporalidad e historicidad, con su existir en el mundo y con la manera como trabaja la comprensión de sí mismo. La narración, como hermenéutica, es un acto de la consciencia reflexiva en el que el sujeto se hace un intérprete de sí mismo (Ricoeur, 2003, 2006).

El asunto de las narrativas se ha posicionado en la filosofía, la lingüística, la teoría literaria y el psicoanálisis como una mediación para hablar del sujeto y de sus procesos de subjetivación (Arfuch, 2008). La fuerza la tiene aquí el reconocimiento de lo experiencial, el testimonio de lo vivido y la narración de primera mano contada por el protagonista de la historia.

El giro subjetivo de esta época está hablando de la cercanía, proximidad y la veracidad del testimonio como fuentes para comprender al sujeto que, por el trabajo narrativo, ha podido dialogar con ese otro que se encuentra inmerso en su intimidad, que emerge gracias al lenguaje y a la experiencia de la otredad que es el reconocimiento de la intersubjetividad que lo constituye. Las narrativas son en últimas una extensión de los enfoques interpretativos en Ciencias Sociales (Escalante, 2013).

Estamos, sin duda, frente a una presencia acentuada de las narrativas que ha tenido su asiento en la literatura y que ha pasado de manera vigorosa a las Ciencias Sociales donde sus usos han sido de particular densidad para ampliar el ámbito de lo biográfico y lo autobiográfico (Rodríguez, 2009).

Las narrativas están situadas en una matriz de investigación cualitativa más cercana al paradigma de la comprensión. El interés de indagación propio de lo cualitativo tiene que ver con la captación y reconstrucción de significados que se van configurando en el mismo proceso narrativo a partir de la experiencia de los mismos actores.

Por medio del enfoque cualitativo, la investigación busca comprender la perspectiva de los participantes, profundizar en sus experiencias de vida, en lo que piensan y en la manera como perciben subjetivamente la "realidad". Desde este marco cualitativo, la investigación con narrativas posibilita que los sujetos cuenten sus propias experiencias y sean reconocidos como protagonistas capaces de comprenderse. Lo que está en la base es un complejo proceso de análisis realizado a varias voces, con intensidades y compromisos diferentes, por parte de los participantes y del investigador.

Las historias de vida, como mediación cualitativa, buscan describir de manera dialéctica el acontecer cotidiano, las tramas del existir, las maneras como los sujetos sobreviven y reconstruyen la vida mediante complejos procesos de interpretación de las situaciones históricas (Chárriez, 2012). 
Las personas van viviendo en la medida en que construyen historias sobre la experiencia de la vida. Esta manera de organizar e integrar los hechos para contarlos tiene que ver con las estrategias que las personas utilizan para construir relatos autobiográficos. Tales estrategias, de alguna manera, condicionan la manera como los narradores, protagonistas, piensan sobre sí mismos, el mundo y sus propias acciones (Duero \& Limón, 2007). Las narrativas son construcciones complejas, configuradas a partir de tramas densas en un ámbito sociohistórico en el que se negocia con otros. Cuando se cuentan historias, los sujetos se hacen protagonistas y pueden entender de una mejor manera su propia existencia, siempre en diálogo, en conversación e interacción con otros (Mangnabosco, 2014).

Connelly y Clandinin (1995), al trabajar los relatos de la experiencia y la investigación narrativa, entienden que el estudio con narrativas tiene que ver con la manera como los sujetos comprenden el mundo: "El estudio de las narrativas, es el estudio de la forma en que los seres humanos experimentamos el mundo" (Connelly \& Clandinin, 1995, p. 11). La narrativa es entendida como el fenómeno mismo que se investiga, pero también como el método utilizado en la investigación. Desde esa lógica, se traza la distinción entre historia, relato y narrativa, llamando historia o relato al fenómeno que se estudia y narrativa a la investigación propiamente dicha. La distinción sutil la plantean en estos términos:

Narrativa es el nombre de la cualidad que estructura la experiencia que va a ser estudiada, y es también el nombre de los patrones de investigación que van a ser utilizados para su estudio (Connelly \& Clandinin, 1995, p. 12).

La investigación narrativa se va tejiendo en un diálogo entre el sujeto que cuenta la historia y el investigador que la escucha atentamente. Entre los dos se va creando una "unidad narrativa compartida" (Connelly \& Clandinin, 1995, p. 18). La investigación con narrativas va configurando una relación colaborativa. Esta experiencia de entrar en contacto con la vida de otro para contarla y relatarla invita a configurar una relación análoga a la amistad, centrada en el respeto y la confianza. De esta manera, la entrevista conversacional, más allá que la respuesta a un cuestionario, se asemeja a una conversación entre amigos dispuestos a escucharse, en búsqueda de la comprensión de la propia vida como relato, de un relato que, como lo expresa bellamente Paul Ricoeur (2006), está en búsqueda de un narrador.

Desde ese horizonte de comprensión, la interpretación desde el trabajo con narrativas pasa también por la interpretación de la propia vida del que está investigando. Comprender e interpretar se encuentran en un solo momento narrativo. Lo que se va mostrando es una convivencia entre el que narra la historia y el que la escucha. La búsqueda del sentido de la historia es un diálogo entre subjetividades que se encuentran para buscar el sentido y los significados de la propia vida (Peña, 2014).

Ahora bien, las narrativas son construcciones que se dan no tanto en el mundo íntimo de las personas, como fruto de su inteligibilidad privada sobre lo vivido, sino también como formas sociales de significación, fruto de procesos intersubjetivos y conversacionales (Gergen, 1996). Esta comprensión dialógica de las prácticas narrativas, como las entiende Gergen (1996), permite reconocerlas en su configuración sociohistórica, como un proceso social dialógico.

Las formas en que los sujetos atribuyen significados a lo que les pasa, afectará, no solo la forma como construyen su identidad, sino también las maneras como estos 
El método de las historias de vida rescata la propia existencia de la subjetividad como forma válida de saber, al poner el acento en la experiencia de los sujetos como actores sociales y en la manera como construyen sus posibilidades de vida digna, desde el agenciamiento de la propia subjetividad vivida en contextos sociales específicos. sujetos actúan en diferentes contextos y cómo se relacionan con los otros. Las narrativas son construcciones sociohistóricas. Por eso, al hacerlas parte del lenguaje, lo que buscan es anunciar nuevas formas de vida, instaurando la esperanza y la consciencia de que otro mundo es posible.

Con todo, las narrativas son formas colectivas que permiten comprender el mundo e inscribir a los sujetos en la historia y en la cultura. Al contar historias, los sujetos están narrando también la época que les ha tocado vivir. Al narrar se hacen participes de una historia común, de la cual pueden sacar sentidos y significaciones (Delory-Momberger, 2015).

\section{Las historias de vida y su significación para la comprensión de la resiliencia como proceso de humanización}

Las investigaciones con historias y relatos de vida se han vuelto muy recurrentes en el ámbito de las Ciencias Sociales, sobre todo cuando se quieren abordar las experiencias límites de los sujetos sometidos a múltiples adversidades (Gómez, 2014). De igual manera se han ido profundizando reflexiones metodológicas y epistemológicas centradas en el análisis de textos que se configuran a partir de las vivencias y acontecimientos de los sujetos, que buscan ser narrados en historias o relatos de vida (Pretto, 2011).

La recurrencia en el trabajo investigativo con historias de vida se presenta, no solo por el declive histórico del positivismo, en su pretensión de absoluta objetividad a partir de datos fríos, sino también por el sugestivo interés por teorizar a partir de la propia vida, afianzando así lo que se ha llamado el "método biográfico", reconociendo a la propia subjetividad como fuente de saber (Cornejo, Mendoza, \& Rojas, 2008).

Las historias de vida tienen su fundamento en el giro narrativo de la investigación social, en el interés renovado de hacer de lo biográfico una fuente privilegiada de conocimiento para la acción y las transformaciones sociales. Volver al sujeto es recuperar a quienes han sido invisibilizados por los poderes hegemónicos que los han subordinado, adquiriendo así las narrativas una dimensión política al permitir espacios democráticos donde los sujetos como ciudadanos sean protagonistas de su propia historia (Sancho Gil, 2014).

El método de las historias de vida rescata la propia existencia de la subjetividad como forma válida de saber, al poner el acento en la experiencia de los sujetos como actores sociales y en la manera como construyen sus posibilidades de vida digna, desde el agenciamiento de la propia subjetividad vivida en contextos sociales específicos.

Frente al tratamiento propio del método científico, de carácter cuantitativo, estadístico y matemático, se ha ido afianzando cada vez más el método biográfico, de los relatos de vida o las historias de vida. Este reivindica la propia experiencia o subjetividad en general como fuente de conocimiento, incluso para las ciencias sociales (Rodríguez, 1999, p. 86).

Para los investigadores en Ciencias Sociales, las experiencias de las personas, recogidas en las historias y los relatos de vida, tienen que ver con la posibilidad de recuperar los sentidos que están presentes en esas historias, muchas veces de forma tácita e implícita. Las historias de vida van reflejando la manera como un grupo humano enfrenta de manera explícita o simbólica la vida, desde sus propias voces (Brandt, 2001). 
En las historias de vida lo singular no es lo individual. Ellas están expresando la existencia "encarnada" de un sujeto, como una co-vivencia con los modos de pensar y concebir la "realidad". La vida del sujeto que aparece en la historia de vida es más bien la historia de la comunidad a la que pertenece, el ámbito de la cultura de la que hace parte expresada desde su voz, como de la lectura que hace de los acontecimientos sociales desde su propia experiencia vital.

Las historias de vida consisten en una exégesis, una explicitación, del acontecer de la vida personal situada en la cotidianidad de un grupo humano, de una comunidad, de una sociedad determinados; desde otro ángulo, los grupos concretos e históricos a los que ha pertenecido y pertenece un sujeto parecen hablar ideográficamente, esto es, con la particularidad de lo que sucede una sola vez en el tiempo, en cada una de las personas con las que se conforma ese grupo humano (Brandt, 2001, p. 14).

Las historias de vida inscritas en las amplias posibilidades metodológicas que hoy existen en el campo de los enfoques biográficos son unas formas de conocimiento que permiten reunir los acontecimientos más significativos de la vida de una persona a partir de la memoria y el recuerdo que aquella percibe como tales. En ellas podemos reconocer trayectorias, caminos recorridos, formas de pensar sobre hechos y situaciones vividas, alegrías y tristezas, fracasos y logros (Carmona, Moreno, \& Tobón, 2012). En ellas podemos también reconocer maneras singulares de afrontamiento de la vida, vínculos o tensiones con personas que han intervenido, lugares especiales que quedan en la memoria, acontecimientos inolvidables que han dejado huella, sistemas de entramados sociales y relaciones profundas, expresadas por cuidados amorosos, o por la existencia de relaciones duras de maltrato, abuso, que han causado sufrimiento.

No obstante, reflejan también una época, un universo situado del que el sujeto ha hecho parte y desde el cual se ha ido configurando en su subjetividad. Ese universo situado según la perspectiva de Brandt es lo que él mismo llama: "Los códigos fundamentales de una cultura" (Brandt, 2001, p. 15), entendidos como aquellos entramados de marcas, huellas y vestigios que están en la base de los pensamientos, las voluntades y los afectos de los sujetos que participan de esa cultura.

Desde la perspectiva de Korniblit (2007), lo individual y personal que narra el sujeto están imbricados y dejan entrever, por un lado, la complejidad de lo social, mostrando cómo los sentidos de vida no están solo en el ámbito de lo individual, sino que están insertados en el contexto social de donde surgen permitiendo un conocimiento más denso del mundo social donde acontece la vida:

Las experiencias particulares de las personas recogidas a través de las historias de vida representan la posibilidad para recuperar los sentidos, vinculados con las experiencias vividas, que se ocultan tras la homogeneidad de los datos que se recogen con las técnicas cualitativas. Pero a la vez plantean el desafío de volver a insertar los sentidos individuales atribuidos a las experiencias en el contexto social en el que ellos surgen, única vía de trascender lo particular y construir un saber más denso sobre lo social (Korniblit, 2007, p. 15).

Las historias de vida permiten reconocer la interpretación que cada narrador de historias le da a su propia vida, desde la legitimidad que le da ser el autor principal de su obra. En contraste con otras mediaciones, como las entrevistas estructuradas, 
Rodríguez (1999) entiende que el objeto de estudio de las historias de vida se va construyendo a lo largo del proceso de investigación. En las historias de vida, lo que se cuenta es una praxis vivida que se apropia de las estructuras sociales, las interioriza y las expresa mediante un relato que cuenta historias. en las que se hacen preguntas similares, en las historias de vida se está más cerca de las entrevistas en profundidad, que tienen un enfoque más conversacional.

La construcción de la historia de vida se va tejiendo entre encuentros conversacionales flexibles y dinámicos, que intencionalmente no quieren dirigir, pues se sitúan en la esencia de la conversación, que es posibilidad de escucha de lo nuevo que surge en un espacio sin restricciones, dando la posibilidad a la aventura de una conversación abierta no directiva, que se permite transitar por caminos inéditos en búsqueda de la construcción del sentido.

Por cuanto subjetividades hechas palabras las historias de vida hacen las veces de encrucijada para el encuentro entre la vida de sus autores, sus investigadores y sus lectores; el desacuerdo interpretativo inherente a cualquier investigación situada epistemológicamente afuera de los vestigios del positivismo y del método hipotético deductivo, proviene de la búsqueda de los temas implícitos en los relatos, de la veracidad de los datos o, en último término, de la fundamentación de los resultados de la investigación no de la experiencia personal narrada en las historias: los acontecimientos, hechos y experiencias difícilmente pueden ponerse en duda si aceptamos y asumimos cada historia de vida en cuanto tal (Brandt, 2001, p. 16).

Rodríguez (1999) entiende que el objeto de estudio de las historias de vida se va construyendo a lo largo del proceso de investigación. En las historias de vida, lo que se cuenta es una praxis vivida que se apropia de las estructuras sociales, las interioriza y las expresa mediante un relato que cuenta historias. El relato del sujeto que colabora con la investigación se va complejizando, por un lado, por la propia lectura del que narra desde el lugar de su memoria, como por la compleja y densa búsqueda de significación donde intervienen los sujetos que interactúan en la investigación (el autor de la narración y el investigador).

Se puede afirmar, siguiendo a Rodríguez (1999), que el estudio de las historias de vida se centra en dos ámbitos fundamentales: la dimensión socio-estructural y la dimensión socio-simbólica. Lo estructural, más referido a lo jurídico, lo económico, lo político, lo cultural; lo simbólico, más en la línea de las representaciones en clave de significaciones y sentidos. Unas y otras ponen en tensión la dialéctica de lo íntimo y lo público, en la esfera del mundo social de la vida.

Desde la perspectiva de Pujadas (2002), las historias de vida tienen que ver con relatos autobiográficos, donde surge la voz del sujeto su testimonio en relación con hechos, situaciones, circunstancias y relaciones que son experimentados como acontecimientos, es decir, algo que se hace significativo por su cualidad para significar un corte, un cambio, una transformación.

Las historias de vida son narrativas realizadas por la propia iniciativa de una persona a partir de unas motivaciones y siguiendo un sistema de elaboración que nos son desconocidos y que hay que intentar averiguar, para poder evaluar su verdadera significación (Pujadas, 1992, p. 48).

Pujadas (1992) reconoce que las historias de vida, como los relatos de vida, pertenecen a unos campos más amplios, como son la biografía y la autobiografía, mediaciones que permiten un acercamiento al sujeto en el ámbito de lo social. Con el advenimiento del método biográfico en las Ciencias Sociales se va reconociendo el 
valor de la vida personal y la capacidad que tiene un investigador para recoger la narrativa vital de un sujeto inmerso en un complejo entorno social. No obstante, entre biografía y autobiografía se pueden anotar algunas diferencias:

La diferencia principal entre ambos términos es que mientras el segundo constituye la narración de la propia vida, contada por su propio protagonista, el primero consiste en una elaboración externa al protagonista, normalmente narrada en tercera persona, ya sea sobre una base documental, ya sea mediante una documentación de documentación, entrevista al biografiado y a otras personas de su entorno (Pujadas, 1992, p. 13).

Según Pujadas (1992) el enfoque biográfico debería constituirse, como método, en algo nuclear del camino cualitativo, ya que permite establecer puntos de encuentro entre el testimonio subjetivo de un individuo a partir de su propia experiencia vital y la expresión de una época, una cultura y unos valores compartidos por la comunidad de la cual el sujeto hace parte.

Ahora bien, al hablar de lo autobiográfico, lo biográfico, de los relatos y las historias de vida se está entrando en un universo de géneros discursivos que buscan, desde diferentes mediaciones, aprehender la propia vida en sus vicisitudes, vivencias y tramas. El carácter multifacético de estas mediaciones pide un ejercicio de diferenciación de lo que cada una está significando. Por ahora, se plantea la diferencia entre "historia de vida" y "relato de vida".

Las historias de vida implican un minucioso rastreo de la trayectoria de vida de un ser humano. En ella, los sujetos que se escogen son ejemplarizantes y se pueden reconocer como prototípicos del asunto que se busca indagar o comprender. La indagación se concentra en entrevistas frecuentes con una sola persona. En las historias de vida se investiga sobre un individuo determinado y se incluye el propio relato, valorando los hechos tal como sucedieron en un recuento cronológico. (Carmona-Parra, 2013).

Al no pretender narrar toda la vida del sujeto, sino algunos acontecimientos particulares, situaciones o hechos significativos, surgen los relatos de vida. Estos relatos ponen de relieve aspectos específicos de gran valor para la vida del sujeto y se van tejiendo desde la manera como estos transitan por sus experiencias vitales.

Los relatos de vida se presentan más como narraciones biográficas enfocadas al interés particular del investigador. La diferencia con las historias de vida estriba en el foco de la mirada, que centra su atención en un aspecto particular de la experiencia. En los relatos de vida se suelen hacer las entrevistas con personas que han caminado por experiencias similares. El relato de vida es más una reflexión de lo social a partir de un relato personal (Korniblit, 2007, p. 15).

Pujadas (1992), en esta misma dirección, plantea también una distinción de fondo y no de forma entre historia de vida y relato. Según este autor, la historia de vida está más centrada en la narración biográfica que hace una persona sobre su propia vida. El relato de vida, que recibe el nombre de "relato biográfico" o "narración", tendrían que ver más con lo que cuenta una persona, pero donde el investigador, apoyándose en el relato que se cuenta, busca, además, otro tipo de información que le permita ampliar o complementar el relato, como serían documentos, fotografías, diarios, colecciones epistolares y archivos que favorecen una reconstrucción objetiva y exhaustiva de lo que se está relatando. 
Franco Ferrarotti (2011), un autor muy reconocido en estos temas, plantea que las historias de vida más allá de ser un método de indagación, son fundamentalmente una apuesta epistemológica que abre nuevas posibilidades al conocimiento en Ciencias Sociales y las califica como una fuente privilegiada de conocimiento social.
Según la perspectiva de Pérez, en su obra Investigación cualitativa: retos e interrogantes (2000), las historias de vida están enmarcadas en un horizonte interpretativo donde los sujetos crean significados:

Las historias de vida ofrecen un valioso marco interpretativo a través del cual el sentido de la experiencia humana se revela en relatos personales de modo que da prioridad a las explicaciones individuales de las acciones más que a los métodos que filtran y ordenan las respuestas en categorías conceptuales predeterminadas. Por medio de ellas el investigador podrá indagar cómo los individuos crean y reflejan el mundo social que los rodea, siendo capaz de captar los procesos y las formas como los individuos perciben el significado de su vida social, pudiendo corroborar el sentido que tiene la vida para ellas (Pérez, 2000, p. 45).

Ruiz (2003), en su trabajo sobre mediaciones cualitativas, identifica algunos objetivos propios de las historias de vida y reconoce que las historias de vida permiten identificar la totalidad de una experiencia en el tiempo y el espacio, captando los rasgos significativos de la vida de esa persona en el ámbito de su ambiente social y familiar. Por otro lado, ayudan en el reconocimiento de la complejidad de la vida, las ambigüedades, las tensiones, las contradicciones experimentadas a lo largo de la existencia y la manera como el sujeto comprende desde sus subjetividades esos tránsitos en el tiempo (Ruiz, 2003, p. 267).

En la historia de vida, según Ruiz (2003), se debe tratar de incluir la mayoría de los datos que puedan modificar cualquier interpretación de la vida del sujeto. Se buscará recoger los cambios más significativos, los hechos, los acontecimientos que en la línea de la vida van marcando un hito o un momento clave digno de ser relatado. Comprender a un sujeto es reflejar la historia de su aparición y su devenir siguiendo el carácter histórico de su acontecer en el mundo. Los datos del sujeto provienen de la vida cotidiana, de la reconstrucción que el individuo mismo hace de su propia vida. Al reconocer los procesos concretos que se pueden identificar y los modos como los individuos captan y crean su vida social, entonces se podrá vislumbrar el significado subjetivo que las cosas tienen para estos sujetos.

Franco Ferrarotti (2011), un autor muy reconocido en estos temas, plantea que las historias de vida más allá de ser un método de indagación, son fundamentalmente una apuesta epistemológica que abre nuevas posibilidades al conocimiento en Ciencias Sociales y las califica como una fuente privilegiada de conocimiento social.

La historia de vida es un texto. Un texto es un "campo", un área más bien definida. Es algo "vivido": con un origen y un desarrollo, con progresiones y regresiones, con contornos sumamente precisos, con sus cifras y su significado. Debo aproximarme a este texto con atención humilde, silenciando al "aventurero interior". Se requiere acercarse al texto con el cuidado y el respeto debido a otro distinto de uno mismo. Se entra en el texto. No basta con leerlo con la atención externa de quien lee solo para informarse. Es necesario "habitarlo" (Ferrarotti, 2011, pp. 107-108).

Según Ferrarotti (2011), la historia de vida es un texto con el que establecemos vínculos y estrategias de liberación. Desde su perspectiva, la historia de vida no es un método o una herramienta metodológica sin más, sino una posibilidad de análisis que permite profundizar en las representaciones y subjetividades particulares, que no es posible conocer por otros medios investigativos. 
La historia de vida se me presenta entonces como una historia de constricciones que pesan sobre el individuo - un conjunto de condicionamientos más o menos determinantes-, y al mismo tiempo como un complejo de estrategias de liberación, que el individuo pone en juego aprovechando las "buenas ocasiones", los atisbos intersticiales (Ferrarotti, 2011, p. 108).

Otro elemento importante que aporta Ferrarotti es el intento de relacionar texto y contexto, buscando vincular las historias de vida con la historia, concebida esta como acontecer social. Ferrarotti (2011) entiende que la historia de vida no es un dato aislado que corresponde a la vida íntima de un sujeto particular, sino que en ella se establece toda una tensión entre la historia del sujeto y sus ligaduras, es decir, con el entramado complejo de órdenes sociales, económicos y culturales donde transita y se encarna la historia de vida.

La historia de vida está inmersa en un contexto en el que se puede reconocer su sentido histórico, evocativo y recreativo y su sentido socio-económico (Ferrarotti, 2011). Lo que está en la base de esta descomposición es la conciencia de entender que se está inmerso en un complejo entramado de relaciones y realidades diversas. Nadie está jamás solo. Solo se existe desde la interdependencia que expresan los encuentros intersubjetivos.

En las historias de vida el sujeto narra a otra persona lo que ha vivido. Ese otro está presente física y actualmente como interlocutor. Los dos entran en diálogo y en la interacción van construyendo la propia historia de vida, que tiene un actor protagónico en el que cuenta la historia y un oyente de excepción en el investigador que la escucha. El investigador acoge el relato y lo trabaja desde su propia lectura, análisis y compresión. La historia de vida es, sin duda, una elaboración compartida, un trabajo conjunto en búsqueda de la comprensión profunda de la vida de los sujetos (García, 2015).

\section{El trabajo con narrativas en resiliencia}

La resiliencia como categoría comprensiva para significar los tránsitos del sujeto en situación de adversidad ha tenido últimamente un desarrollo significativo. (GarcíaVesga \& Domínguez de la Ossa, 2013). En este proceso el diálogo con las narrativas se ha hecho muy significativo. El trabajo con las narrativas desde las historias o relatos de vida es hoy una mediación metodológica potente para comprender procesos de subjetivación, ya que permite reconocer las maneras como los sujetos reconstruyen su vida desde el mismo trabajo narrativo. (Aya, 2010). La construcción del relato posibilita que los sujetos se reinventen en el presente. Esto hace coherencia con la mirada de la resiliencia, que le apuesta a la posibilidad y capacidad de tránsitos en el sujeto del padecimiento, el dolor y el sufrimiento, hacia el reencuentro con una nueva vida pasada por la memoria y la imaginación:

La desgracia nunca es algo puro, tampoco la felicidad. Pero apenas la convertimos en relato, damos un sentido al sufrimiento y comprendemos, mucho tiempo después, cómo pudimos transformar una desgracia en maravilla, ya que todo hombre herido se ve forzado a la metamorfosis: aprendí a transformar la desgracia en una prueba. Si una te baja la cabeza, la otra te la levanta (Cyrulnik, 2001, p. 10).

Gracias al poder evocador y creativo de las narrativas, el sujeto no se queda anclado en "lo que pasó", en el horror de la herida, en el hecho duro y doloroso de la adver- 
Pág 13

No es posible hablar de resiliencia si no existe una narrativa de afrontamiento y transformación. No es posible hablar de resiliencia si no existe un relato que permita la reconfiguración de la vida y la afectación de las condiciones sociales, económicas, culturales y políticas que empobrecen, explotan y marginan a los sujetos y a las comunidades. sidad que ha hecho daño y que sigue doliendo, sino que transita hacia algo nuevo, haciendo de su sufrimiento un relato, es decir, una elaboración de la propia vida como posibilidad de ser en medio del caos, del hecho adverso, de la situación límite (Cyrulnik, 2001). La resiliencia se refiere a la capacidad de los sujetos para sobreponerse y salir adelante fortalecidos. El anclaje ahora es con "lo que hace el sujeto con lo que le pasó". Se refiere esto a la búsqueda de sentido y significación que hace de la historia presente una oportunidad para reinventar el futuro con esperanza.

Es por esto que la mayoría de autores trabajan la categoría de resiliencia a partir de la narración de una multitud de relatos y de historias de vida de sujetos en diferentes contextos, países y circunstancias, en las que se pueden reconocer las maneras como estos sujetos y comunidades construyen en la práctica la resiliencia. En esos relatos, lo íntimo transita hacia lo público, desde una acción comprometida que trabaja por una vida digna y justa.

La propia naturaleza de la resiliencia la define como respuesta dinámica, no estática, a la vez que creativa, en la que adquiere especial importancia la capacidad de construir nuevas interpretaciones de los contextos adversos y de las respuestas posibles que todos podemos encontrar ante ellos. Tenemos en cuenta que en definitiva un proceso que podríamos llamar resiliente representa, entre otras cosas, la elaboración de una nueva interpretación de los acontecimientos y de los contextos y, por ende, una nueva mirada de las personas (Mandariaga, Palmas, Surjo, Villalba, \& Arribillaga, 2014, p 12).

No es posible hablar de resiliencia si no existe una narrativa de afrontamiento y transformación. No es posible hablar de resiliencia si no existe un relato que permita la reconfiguración de la vida y la afectación de las condiciones sociales, económicas, culturales y políticas que empobrecen, explotan y marginan a los sujetos y a las comunidades.

La resiliencia reconoce que la vida está tocada por el "poder" evocador del relato, por su fuerza transformadora y constructora de nuevas realidades. El acontecer en el mundo no se puede separar de un inicio "pre-narrativo" que lo constituye. Toda existencia humana, en medio de la precariedad del existir, reclama un nuevo comienzo desde un relato evocador.

El tercer anclaje del relato en la vida consiste en lo que se podría llamar la cualidad pre-narrativa de la experiencia humana. Es gracias ella que tenemos el derecho a hablar de la vida como una historia en estado naciente y, por lo tanto, de la vida como una actividad y una pasión en busca de relato (Ricoeur, 2006, p. 18).

Los sujetos se configuran desde una estructura pre-narrativa de la experiencia (Ricoeur, 2006). Esta estructura está jalonando el ser inacabado del sujeto. Nada queda escrito para siempre. Así como la felicidad no es algo permanente, tampoco lo es la desgracia ni la desdicha. La vida ha demostrado su capacidad para el crecimiento, de esto dan testimonio los relatos. La comprensión narrativa del sujeto se logra en el desplazamiento de lo vivido a lo narrado. Narrar significa en medio del caos y las complejidades del vivir, ir buscando cierto orden a partir del trabajo narrativo, un orden que arma la trama para contar lo vivido e interpretarlo (Escalante, 2013).

Las narrativas se han convertido, para los investigadores en resiliencia, en un referente básico para la comprensión de ella, tanto en sus fundamentos teóricos, como 
Pág 14

La investigación en resiliencia si bien acentúa relatos de sujetos particulares, está entendiendo las conexiones que tienen esos relatos en la construcción de un nosotros colectivo. No es posible afirmar la propia subjetividad sin la intersubjetividad. Por tanto, las historias de vida no son un dato del sujeto individual, sino que están expresando al colectivo, que se puede llamar grupo, clase, generación o época. en su enfoque metodológico a la hora de trabajar con sujetos en situación de adversidad (Manciaux, 2003; Brooks \& Goldstein, 2004; Barudy \& Dantagnan, 2005; Henderson, 2006; Cyrulnik, 2005, 2009; Mandariaga, 2014). Para estos autores, las narrativas están evidenciando procesos de trasformación, en la medida en que los sujetos no solo van contando la historia desde su afrontamiento; sino que van relatando los procesos de agenciamiento de la vida desde la transformación, el aprendizaje y el crecimiento, que va más allá de la sola resistencia a las dificultades.

Esto se puede observar en dos obras clásicas: La felicidad es posible: despertar en niños maltratados la confianza en sí mismos: construir la resiliencia (Vanistendael \& Lecomte, 2002) y La maravilla del dolor: el sentido de la Resiliencia (Cyrulnik, 2001). En los dos textos los investigadores cuentan una variedad de historias y relatos de vida de seres humanos que transitan por situaciones adversas y desgarradoras. Lo interesante es poder rastrear aquí las narrativas resilientes presentes en los procesos de elaboración de la propia vida. La identidad del sujeto se recrea en la posibilidad que tiene el sujeto de contar la vida, en la manera como su yo se reconfigura al contar los relatos. En estos autores el recurso de las narrativas tiene una doble configuración: como experiencia de vida en la construcción de subjetividad y como metodología que reconoce en las narrativas una mediación única para testimoniar lo que han llamado procesos resilientes.

Ahora bien, la investigación en resiliencia si bien acentúa relatos de sujetos particulares, está entendiendo las conexiones que tienen esos relatos en la construcción de un nosotros colectivo. No es posible afirmar la propia subjetividad sin la intersubjetividad. Por tanto, las historias de vida no son un dato del sujeto individual, sino que están expresando al colectivo, que se puede llamar grupo, clase, generación o época. La singularidad no es aquí individualidad o solipsismo, sino un rasgo atravesado por la cualidad de saberse inmerso en un grupo y una comunidad con la que se establecen vínculos, relaciones y responsabilidades éticas y políticas.

La resilencia, como categoría de análisis de lo humano en situación de adversidad, se inscribe en esos procesos comprensivos donde las narrativas ocupan un papel fundamental al favorecer nuevas lecturas y significaciones desde la apropiación de la vida por parte de los protagonistas. Los sujetos, al narrar sus historias, lo que están haciendo es reescribir sus propios guiones de vida (Brooks \& Goldstein, 2004).

Los sujetos que se adentran en el juego de las narrativas desde la mirada resiliente tienen la posibilidad de reorganizar significados, sentidos y prácticas de vida incluso desde su infancia. Este es el caso que ejemplarizaron las investigadoras Sara Victoria Alvarado y Patricia Granada (2010) al trabajar resiliencia con niños y niñas de la calle en Pereira, Colombia. Según su investigación, los niños y las niñas pudieron reencontrarse en su posibilidad de existir de otra manera en el acto mismo de narrarse desde un acontecer resiliente (Alvarado \& Granada, 2010). Las investigadoras, desde un análisis descriptivo, de construcción de sentido e interpretativo, recogieron las narrativas de esos niños y niñas de la calle, reconociendo en ellas no solo experiencias vitales, sino categorías sociopolíticas que mostraron los detonantes de la acción política en esos niños y niñas para sobrevivir en contextos de adversidad, atribuyéndole significados nuevos a su existencia (Alvarado \& Granada, 2010).

Otro trabajo significativo en el uso de las narrativas es la tematización de su lugar en relatos de resiliencia con familias. Según esta investigación, la familia se configura en su resiliencia desde el relato que ella hace de sí en medio de tensiones y conflic- 
tos. Las narrativas permiten elaborar procesos resilientes desde una autorrestauración y crecimiento. El relato fortalece una comprensión sistémica de la familia, que es capaz de rehacerse y actuar en medio de la adversidad de forma interdependiente y solidaria (Builes \& Bedoya, 2008).

Un ejercicio subjetivo que radicaliza el trabajo con narrativas y resiliencia, se relaciona con las narrativas de la Shoah (Holocausto judío). El investigador en resiliencia Jesús Camarero (2014) realiza un estudio de testimonios de tres psicoanalistas que tematizan la experiencia del Holocausto desde el "trauma y la memoria" (H. Parens), la "identidad y trauma" (Viktor Frankl) e "identidad y memoria" (Boris Cyrulnik). Lo novedoso de su trabajo es intentar desarrollar una teoría de la resiliencia fundamentada en los trabajos de Cyrulnik y en el acercamiento a las reflexiones que sobre la función narrativa ofrece Paul Ricoeur (2009) desde una hermenéutica filosófica.

Estos trabajos son coherentes con la afirmación de Daniel Rodríguez (2003), investigador latinoamericano que sostiene que la narrativa es un modo privilegiado de socialización en resiliencia, ya que en ella se pueden reconocer las historias familiares, sociales y políticas, que dan testimonio de la manera como los sujetos se van haciendo resilientes a nivel personal, familiar y comunitario.

El relato de un cuento, un mito, un acontecimiento histórico y social nos pone en comunidad, tanto en el eje del tiempo como del espacio; a su vez somos productores y generadores de nuevas historias (Rodríguez, 2003, p. 111).

Tener la capacidad de generar nueva historia, que es lo propio de la resiliencia como proceso de humanización, pone en la mesa de discusión la necesidad de seguir profundizando la relación entre narrativa y resiliencia, potenciando un diálogo desde la perspectiva ontológica y fenomenológica. El relato permite transitar del padecimiento y del dolor profundo al reconocimiento de entender que la vida es una promesa, donde es posible un nuevo nacimiento (Rodríguez, 2006). Las historias que se cuentan no son solo fruto de un recuerdo o de una memoria del pasado, son en sí mismas un acto configurativo, una creación nueva que acontece cuando el sujeto es capaz de re-interpretarse al contar la experiencia como acontecimiento vital que ha golpeado la vida, pero que no ha mutilado el sentido.

En este punto, es muy iluminador y sugestivo para ese diálogo entre resiliencia y narrativa acoger la perspectiva de Paul Ricoeur y su comprensión de la "Identidad narrativa", que ha sido trabajada fundamentalmente en dos de sus obras clásicas: Tiempo y Narración III. Tiempo narrado (2009) y en Sí mismo como otro (2008). En estos textos, Ricoeur, centrado en la analítica de la temporalidad, el tiempo narrativo y la función configurativa de la trama en el relato de una vida, entiende la identidad como una categoría de la vida práctica, reconociendo al autor como alguien que es capaz de construir su identidad precisamente al narrar la historia de su propia vida:

Identidad está tomada aquí en el sentido de una categoría de la práctica. Declarar la identidad de un individuo o de una comunidad es responder a la pregunta: ¿Quién ha hecho tal acción? ¿Quién es el agente, el autor? Hemos respondido a esta pregunta nombrando a alguien, designándole por su nombre propio... responder a la pregunta ¿quién?, como lo ha hecho Hannah Arendt, es contar la historia de una vida. La historia narrativa dice el quién de la acción, por lo tanto, la propia identidad del quién no es más que una identidad narrativa (Ricoeur, 2009, p. 997). 
Pág 16

Las historias de vida tendrían el "poder" de favorecer procesos de subjetivación y de agenciamiento de la propia vida. Es aquí donde la mediación adquiere fuerza política, dada su capacidad para liberar desde las narrativas, sus posibilidades de construcción de subjetividad, permitiendo a los sujetos liberar su palabra y su poder emancipatorio mediante la fuerza narrativa que permite transformar, reinventar y rehacer la vida herida para la esperanza de un nuevo renacer.
El sujeto que narra y que se narra en el relato es ante todo agente, sujeto de experiencia, que se hace sujeto en el acto de narrar y contar historias. El sujeto desde la mirada de Ricoeur (2009) se va configurando a partir de las diversas formas de asunción del yo, posibilitando a su vez la escucha de otras voces, que abren nuevos espacios para las interacciones sociales, la búsqueda de valores compartidos y de nuevos sentidos, que favorecen la configuración de la comunidad y la democracia (Arfuch, 2010).

Si para la resiliencia el sujeto es un ser que se hace humano al narrar su historia, desde la perspectiva de Ricoeur el sujeto es un agente, un hablante y un sujeto responsable que deja entrever la dimensión ética y política de su ser histórico precisamente en ese mismo acto de narrarse (Ricoeur, 2006).

Si bien Ricoeur (2008) se centró más en las formas literarias de la narración, propias del ámbito de la cultura, no se puede desconocer la significación de la noción de configuración narrativa aplicable a los sujetos y a las maneras como se cuentan las historias en el seno de la acción cotidiana. Es en este horizonte de una identidad construida desde las narrativas donde los sujetos se interpretan a sí mismos, siendo ya no autores de la existencia vivida, sino coautores en la búsqueda del sentido (Escalante, 2013).

El poder evocador de la narrativa, su capacidad para rehacer el mundo humano de la acción, sus capacidades para prefigurar, configurar y refigurar la propia historia contada harían muy interesante un acercamiento dialógico con la resiliencia y la manera como esta entiende los procesos de construcción de subjetividad desde el trabajo narrativo.

\section{Conclusiones}

La historia de vida es una mediación privilegiada para contener las narrativas que se van configurando alrededor de los procesos de tensión y de crisis, en situaciones de adversidad. Ellas, por su carácter dinámico, permiten reconocer las trayectorias de una vida y las maneras como los sujetos van significando los acontecimientos de su existencia. Reconociendo no solo la manera como los sujetos enfrentan realidades, sino el impacto de sus acciones en los grupos sociales de los que hacen parte y a los que pueden influir con sus acciones y decisiones.

Los tránsitos de una vida, los ires y venires del camino, las maneras como se "resiliencia" la vida, los procesos de respuesta y transformación frente a la adversidad tienen en las historias de vida una mediación única para ser reconocidas. Allí se acentúa la fuerza de la voz del sujeto como protagonista, explorando sus emergencias, las líneas de fuga y las posibilidades para agenciar procesos de humanización, liberación y resistencia. Desafiando a aquellas que podrían tender a naturalizar la exclusión y marginalidad, regulando la vida y controlándola por poderes hegemónicos.

Las historias de vida tendrían el "poder" de favorecer procesos de subjetivación y de agenciamiento de la propia vida. Es aquí donde la mediación adquiere fuerza política, dada su capacidad para liberar desde las narrativas, sus posibilidades de construcción de subjetividad, permitiendo a los sujetos liberar su palabra y su poder emancipatorio mediante la fuerza narrativa que permite transformar, reinventar y rehacer la vida herida para la esperanza de un nuevo renacer. En este punto, la historia de vida articula las fronteras entre lo íntimo y lo público, lo adverso y la respuesta, 
tendiendo a complejizar la respuesta de una vida que se enraíza en el mundo para transformarlo desde la solidaridad.

La labor interpretativa de las historias de vida adquiere un valor particular para la resiliencia, pues posibilita reconocer los tránsitos de una vida al enfrentar vicisitudes personales y sociales, como las formas en que se instaura como sujeto social, capaz de acoger, asumir y enfrentar con creatividad y solidaridad las adversidades que están inmersas en contextos de violencia, explotación y abuso del poder.

En este sentido, más allá de un excesivo testimonialismo que pueden encerrar las historias de vida, al poner la fuerza del relato en el sujeto individual, es necesario tensionar más el sentido contextual e histórico, que sitúa al relato de la historia de vida entre las fuerzas de las dinámicas sociales y de las acciones políticas que buscan reinventar la vida. Las historias de vida están jalonando el compromiso político de una vida que se lee en prácticas de agenciamiento y transformación, más allá de las circunstancias y realidades individuales.

Al investigar en resiliencia con historias de vida, es inevitable encontrarse con lo que se puede llamar una unidad narrativa compartida. Tanto el investigador, como los sujetos que relatan historias, están vinculados en una única unidad narrativa mediada por la escucha respetuosa de las diferentes voces y por el cuidadoso proceso de negociación en búsqueda del significado de la experiencia. Resiliencia y narrativas están íntimamente unidas. El proceso resiliente se configura en la medida en que se es capaz de comprender la historia, a medida que se avanza el proceso de investigación. Aquí no solo se cuenta la historia del que relata, sino aquella que es contada por el que trabaja con los relatos.

\section{Referencias}

Alvarado, S. (2014). Ampliación de la comprensión de los procesos de configuración de subjetividades políticas de niños, niñas y jóvenes en Colombia desde una perspectiva alternativa del desarrollo humano: tránsitos y aprendizajes. (17-53). En S. Alvarado \& H. Ospina. Socialización política y configuración de subjetividades. Construcción social de niños, niñas y jóvenes como sujetos políticos. Bogotá. Siglo del Hombre Editores. http://libreriasiglo.com/educacion-y-pedagogia/12346-socializacion-politica-y-configuracion-de-subjetividades-construccion-social-de-ninos-ninas-y-jovenes-como-sujetos-politicos.html\#. WP9AnYiGPIU

Alvarado, S., \& Granada, P. (2010). Resiliencia y sentido político en niños y niñas en situación de calle. Revista Latinoamérica de Ciencias Sociales, Niñez y Juventud, 8(1), 311-327. http://revistaumanizales.cinde.org.co/index.php/Revista-Latinoamericana/article/view/56/15

Arfuch, L. (2010). El espacio biográfico: dilemas de la subjetividad contemporánea. Buenos Aires: Fondo de Cultura Económica. http://www.comisionporlamemoria. org/investigacionyensenanza/materiales/volumen13/docs/1-arte-y-politica/ Texto\% $205 . p d f$

Arfuch, L. (2010a). Sujetos y narrativas. Centros de Estudios Sociológicos. Universidad Nacional Autónoma de México. Acta Sociológica, 53, 19-41. http://www. journals.unam.mx/index.php/ras/article/view/24297/22831

Arfuch, L. (2008). El espacio teórico de las narrativas: un desafío ético y político. Revista internacional de filosofía Iberoamericana y teoría social, 13(42), 131-140. http://www.redalyc.org/articulo.oa?id=27904208 
Aya, S. (2010). Reflexiones acerca de los procesos incluidos en la construcción narrativa. ¿Cómo emergen los reatos? Perspectiva Psicológica, 6(1), 185-194. http://www. scielo.org.co/scielo.php?script=sci arttext\&pid=S1794-99982010000100015

Barudy, J., \& Dantagnan, M. (2005). Los buenos tratos a la infancia: parentalidad, apego y resiliencia. Barcelona: Gedisa. https://www.casadellibro. com/libro-los-buenos-tratos-a-la-infancia-parentalidad-apego-y-resilienci-a/9788497840910/1014631

Brant, J. (2001). Historias de vida una apuesta Epistemológica. Revista Heterotopia, 7(17), 13-39.

Brooks, R., \& Goldstein, S. (2004). El poder de la resiliencia: cómo lograr el equilibrio, la seguridad y la fuerza interior para vivir en paz. Barcelona: Paidós. https://www.casadellibro.com/libro-el-poder-de-la-resiliencia-como-lograrel-equilibrio-la-segurid-ad-y-la-fuerza-interior-necesarios-para-vivir-enpaz/9788449316043/986315

Bruner, J. (1997). La educación, puerta de la cultura. Madrid: Visor Dis. https://www.casadellibro.com/libro-la-educacion-puerta-de-la-cultura/9788477741787/2070149

Bruner, J. (2003). La fábrica de historias: derecho, literatura, vida. México: Fondo de Cultura Económica. https://www.casadellibro.com/libro-la-fabrica-de-historias-derecho-literatura-vida/9789505579501/2348589

Builes, M., \& Bedoya, M. (2008). La familia contemporánea: relatos de resiliencia y salud mental. Revista Colombiana de Psiquiatría, 37(3), 344-354. http://www. scielo. org.co/pdf/rcp/v37n3/v37n3a05.pdf

Camarero, J. (2014). Resiliencia y narrativas de la Shoah: los testimonios resilientes y la práctica neuropsiquiátrica. Revista de Estudios Franceses, 10, 75-93. https:// cedille.webs.ull.es/10/05camarero.pdf

Carmona, J., Moreno, F., \& Tobón, J. (2012). La carrera de las niñas soldado en Colombia: un estudio desde el punto de vista del agente. Medellín: Fondo Editorial Funlam. http://www.funlam.edu.co/uploads/fondoeditorial/preliminar/2012/ La-carrera-de-las-ninas-en-los-grupos-guerrilleros.pdf

Carmona-Parra, J. (2013). Las niñas de la guerra en Colombia. Manizales: Fondo Editorial UCM. http://repositorio.ucm.edu.co:8080/jspui/bitstream/handle/10839/683/ Las\%20Ninas\%20de\%20la\%20Guerra\%20Final.pdf?sequence=1

Chárriez, M. (2012). Historias de vida: una metodología de investigación cualitativa. Revista Griot, 5(1), 50 -67. https://www.google.com.co/url?sa=t\&rct=j\&q=\&es$r c=s \&$ source $=$ web \&cd =1 \&ved $=0$ ahUKEwiiheeS1 $r 3$ TAhUKSSYKHa5jAIOQFggiMAA\&url=http\%3A\%2F\%2Frevistas.upr.edu\%2Findex.php\%2Fgriot\%2Farticle\%2Fdownload\%2F1775\%2F1568\&usg=AFQjCNEeX9ZdLkDtMkndGh6 Al5nQ4v71A

Connelly, F. M., \& Clandinin, D. J. (1995). Relatos de experiencia e investigación narrativa. En J. Larrosa et al., Déjame que te cuente. Ensayos sobre narrativa y educación. Barcelona: Laertes. https://www.google.com.co/url?sa=t\&rct=$j \& q=\& e s r c=s \&$ source $=$ web \& $c d=2 \& v e d=0$ ahUKEwjbwon $-z b$ TAhWDPCYKHRloBJkQFggnMAE\&url=http\%3A\%2F\%2Finstitucional.us.es\%2Frevistas\%2Fcuestiones\%2F13\%2Fart 22.pdf\&usg=AFQjCNEjHgCndOW1 huRbko51RIDJyD54tQ

Cornejo, M., Mendoza, F., \& Rojas, R. (2008). La investigación con relatos de vida: pistas y opciones del diseño metodológico. Revista Psyke, 17(1), 29-39. http://www. scielo.cl/pdf/psykhe/v17n1/art04.pdf

Cyrulnik, B. (2001). La Maravilla del dolor. El sentido de la resiliencia. Barcelona: Granica. http://www.gandhi.com.mx/la-maravilla-del-dolor-el-sentido-de-la-resiliencia 
Cyrulnik, B. (2005). El amor que nos cura. Barcelona: Gedisa. http://www.gedisa.com/ ficha.aspx?cod=100560\#.WP49 YiGPIU

Cyrulnik, B. (2009). Los patitos feos. La resiliencia: una infancia infeliz no determina la vida. Barcelona: Gedisa. http://bibliotecaparalapersona-epimeleia.com/greenstone/collect/libros1/index/assoc/HASH01e0.dir/doc.pdf

Delory-Momberger, C. (2015). La condición biográfica: ensayos sobre el relato en sí en la modernidad avanzada. Medellín: Editorial Universidad de Antioquia. http:// www2.udea.edu.co/webmaster/editorial/fichas-libros/la-condicion-biografica. pdf

Duero, D., \& Limón, G. (2007). Relato autobiográfico e identidad personal. Un modelo de análisis narrativo. Revista de Antropología Iberoamericana, 2(2), 232-275. Recuperado de https://www.researchgate.net/publication/28183315 Relato autobiogrfico e identidad personal Un modelo de anlisis narrativo

Escalante, E. (2013). La perspectiva ricoeuriana y el análisis de las narrativas. Revista Fundamentos de Humanidades, 14(1), 175-192. http://fundamentos.unsl.edu. ar/pdf/articulo-27-175.pdf

Ferrarotti, F. (2011). Las historias de vida como método. Revista Acta Sociológica 56, 95-118. http://www.redalyc.org/pdf/105/10504402.pdf

García, V. (2015). La creación del sí mismo. Revista Mexicana de investigación educativa, 14, 175-192. http://www.redalyc.org/pdf/140/14032722010.pdf

García-Vesga, M.C., \& Domínguez-De la Rosa, E. (2013). Desarrollo teórico de la resiliencia y sus aplicaciones en situaciones de adversidad: una revisión analítica. Revista Latinoamericana de Ciencias Sociales, Niñez y juventud 11(1), 63-77. http:// revistalatinoamericanaumanizales.cinde.org.co/wp-content/uploads/2013/03/ Desarrollo te\%C3\%B3rico a03v111.pdf

Gergen, K. (1996). Realidades y relaciones: aproximaciones a la construcción social. México: Editorial Paidós. https://www.casadellibro.com/libro-realidades-y-relaciones-aproximacion-a-la-construccion-social/9788449303036/539811

Gómez, J. (2014). Memoria, historias de vida y papel de la escucha en la transformación subjetiva de victimas/sobrevivientes del conflicto armado colombiano. El Ágora USB, 14, 37-60. http://revistas.usb.edu.co/index.php/Agora/article/ view/119/78

Henderson, E. (2006). La resiliencia en el mundo de hoy: cómo superar la adversidad. Barcelona: Gedisa. https://www.casadellibro.com/libro-la-resiliencia-en-el-mundo-de-hoy-como-superar-la-adversidad/9788497841382/1107426

Korniblit, A. (Coord.). (2007). Metodologías cualitativas en Ciencias Sociales. Buenos Aires: Biblos. http://biblio.fcedu.uner.edu.ar/derecha/novedades/pdf/17954.pdf

Kotliarenco, M., Cáceres I., \& Fontecilla, M. (1997). Estado del arte en resiliencia. Washington: Organización Panamericana de la Salud. http://www1.paho.org/hq/dmdocuments/2009/Resil6x9.pdf

Loureiro, A. (1991). Problemas teóricos de la autobiografía. Revista Anthropos, 29, 2-9. https://es.scribd.com/doc/158850759/Problemas-teoricos-de-la-autobiografia

Manciaux, M. (2003). La resiliencia: resistir y rehacerse. Barcelona: Gedisa. http:// www.gedisa.com/ficha.aspx?cod=100549\#.WP50cliGPIU

Mandariaga, J. (Coord). (2014). Nuevas miradas sobre la resiliencia: ampliando ámbitos y prácticas. Barcelona: Gedisa. http://www.gedisa.com/ficha.aspx?cod=100618\&titulo=Nuevas - miradas-sobre-la-resiliencia\#.WP50qYiGPIU

Mandariaga, J., Palma, M., Surjo, P., Villalba, C., \& Arribillaga, A. (2014). La construcción social de la resiliencia. En J. Mandariaga (Coord). Nuevas miradas sobre la resiliencia: ampliando ámbitos y prácticas. Barcelona: Gedisa. http://www.gedisa.com/ficha.aspx?cod=100618\&titulo=Nuevas-miradas-sobre-la-resiliencia\#. WP9BV4iGPIU 
Magnabosco, M. (2014). El construccionismo social como abordaje teórico para comprender el abuso sexual. Revista de Psicología, 32(2), 220-242. http://www.redalyc.org/articulo.oa?id=337832618002

Peña, J, (2014). La vida exige ser narrada. Anuario Filosófico, 47(3), 567 -587. http:// proyectoscio.ucv.es/wp-content/uploads/2016/03/02-Estudios Penya Vial.pdf

Pérez, G. (2000). Investigación cualitativa: retos e interrogantes. Madrid: Muralla. https://www.casadellibro.com/libro-investigacion-cualitativa-i-retos-e-interrogantes--metodos-6-ed/9788471336286/390717

Pretto, A. (2011). Analizar las historias de vida: reflexiones metodológicas y epistemológicas. Revista Tabula rasa, 15, 171-194. http://www.scielo.org.co/pdf/tara/ n15/n15a10.pdf

Puig, G., \& Rubio, L. (2011). Manual de resiliencia aplicada. Barcelona: Gedisa. http:// www.gedisa.com/ficha.aspx?cod=100609\&titulo=Manual-de-resiliencia-aplicada\#.WP5PloiGPIU

Pujadas, J. (1992). El método biográfico: el uso de las historias de vida en ciencias sociales. Cuadernos Metodológicos 5. Madrid: CIS (Centro de Investigación Sociológica). http://www.cis.es/cis/opencm/ES/3 publicaciones/colecciones/ver. isp?id $=9788474761740$

Ricoeur, P. (2003). Teoría de la interpretación. Discurso y excedente de sentido. México: Siglo XXI editores. http://mastor.cl/blog/wp-content/uploads/2015/08/ Ricoeur.-Teoria-de-la-interpretacion.-Siglo-XXI.pdf

Ricoeur, P. (2006). La vida un relato en busca de narrador. Revista Ágora, papeles de filosofía, 25(2), 9-22. http://perio.unlp.edu.ar/catedras/system/files/ricoeur-lavida-un-relato-en-busca-de-narrador.pdf

Ricoeur, P. (2008). Si mismo como otro. Madrid: Siglo XXI Editores. https://construcciondeidentidades.files.wordpress.com/2014/08/ricoeur-paul-si-mismo-como-otro.pdf

Ricoeur, P. (2009). Tiempo y Narración III. Tiempo narrado. México: Siglo XXI Editores. https://textosontologia.files.wordpress.com/2012/11/tiempo-y-narracic 3b3n-iii.pdf

Rodríguez, D. (2003). Resiliencia, subjetividad e identidad. Los aportes del humor y la narrativa. En A. Melillo, \& D. Rodríguez (comps.). Resiliencia y subjetividad: los ciclos de la vida. Barcelona: Paidós. https://www.casadellibro.com/libro-resiliencia-y-subjetividad-los-ciclos-de-la-vida/9789501245301/1019345

Rodríguez, J. (1999). El Método de las historias de vida. Revista Antropos, 20-1(38), 85-101.

Rodríguez, C. (2006). Narrativas resilientes en policías discapacitados por hechos violentos. Pensamiento Psicológico, 2(7), 41-53. http://www.redalyc.org/articulo. oa?id=80120704

Rodríguez, J. (2009). Asedio a las narrativas contemporáneas. Mapa de posibles investigaciones. Cuadernos de Literatura, 14(26), 14-51. http://revistas.javeriana. edu.co/index.php/cualit/article/view/6309/5052

Ruiz, J. (2003). Metodología de la Investigación Cualitativa. Deusto: Universidad de Bilbao. https://es.scribd.com/document/250867128/Metodologia-de-la-Investigacion-Cualitativa-JOSE-IGNACIO-RUIZ-OLABUENAGA-2012-pdf

Sancho Gil, J. (2014). Historias de vida: el relato biográfico entre el autoconocimiento y dar cuenta de la vida social. Revista Praxis Educativa, 18(2), 24-33. http://www. scielo.org.ar/scielo.php?script=sci arttext\&pid=S0328-97022014000200003

Vanistendael, S., \& Lecomte, J. (2002). La felicidad es posible. Despertar en niños maltratados la confianza en sí mismos: Construir la resiliencia. Barcelona: Gedisa. $\quad$ https://www.casadellibro.com/libro-la-felicidad-es-posible-despertar-en-ninos-maltratados-la-confia-nza-en-si-mismos-construir-la-resiliencia/9788474329650/854377 